\title{
BIT ERROR RATE ANALYSIS OF WIMAX PHYSICAL LAYER UNDER SUI AND AWGN CHANNEL MODEL WITH VARYING CYCLIC PREFIX, MODULATION TECHNIQUES AND CODING SCHEMES
}

\author{
Saurabh Kher ${ }^{1}$, Sh. Gurpadam Singh ${ }^{2}$ \\ ${ }^{1}$ Assistant Prof, E.C.E, GNDU RC, Gurdaspur \\ ${ }^{2}$ Associate Prof. E.C.E, BCET Gurdaspur
}

\begin{abstract}
Requirement of portable, mobile and high speed connectivity for internet access is increasing day by day. WiMAX will be playing an important role in the Broadband Wireless Technology (BWT) sector, since it is more cost-effective and easy to install technology. It is a fixed Broadband Wireless Access (BWA) system based on the IEEE 802.16 standards and will be used to provide "last mile" access to the broadband and Internet access services. The WiMAX technology is based on the IEEE 802.16-2004 Air Interface Standard and is rapidly proving itself as a technology that will play a significant role in fixed broadband wireless metropolitan area networks. It is based on the concept of orthogonal frequency division multiple access technique in wireless communication. This paper investigates the performance of WiMAX network under AWGN and SUI Channel Model by varying physical layer parameter such as higher M-ary modulation, coding scheme and cyclic prefix.
\end{abstract}

Keywords: BWA, BWT, WiMAX, OFDM, CP, WLAN

\section{INTRODUCTION}

Fifteen years back very few people have thought that internet will become the backbone of the society. It has justified its presence when we see that internet will become integral part of our life. It has revolutionalised our lives to such an extent that things which we would be able to do in days are now possible at a click of a button. Today life is changing at the rapid pace, thanks to the evolution of telecommunication industry. So internet, broadband and mobile technology has become the part of our daily life now a day. The advancement of wireless communication technology provides the advantages of wide area coverage without wires and allowing portability and mobility while communicating. This is only due to the success of mobile telephony which leads to success of new wireless communication services [1].

WiMAX (Worldwide Interoperability for Microwave Access) is based on the IEEE 802.16 standard for Metropolitan Area Networks (MAN). Its goal is to deliver wireless broadband access to customers using base stations with coverage range up to few miles. The IEEE WiMax/802.16 is a promising technology for broadband wireless metropolitan area networks (WMANs) as it can provide high throughput over long distances and can support different qualities of services [2]. The IEEE $802.16 \mathrm{~m}$-based WiMAX 2.0 system is providing transmission rate upto $1 \mathrm{~Gb} / \mathrm{s}$. The most important objective of this paper is to utilize wider channel bandwidth. Additionally, this technology utilizes Multicarrier technique to utilize wider bandwidth for parallel data transmission across multiple RF carriers. The IEEE 802.16 wireless technology can be an excellent choice for Cellular/Mobile applications due to its high bandwidth and long range. Features of this technique will be summarized as under:

(a) It is cost effective.

(b) It offers high data rates.

(c) It supports fixed, nomadic and mobile applications.

(d) It is easy to deploy and has flexible network architectures.

(e) It supports interoperability with other networks.

All three standards such as $802.16,802.16 \mathrm{a}$ and $802.16 \mathrm{c}$ were merged together and a new standard was created which is known as 802.16e. In the beginning, it was published [4] as a revision of the standard under the name $802.16 \mathrm{REVd}$, and due to its popularity finally given the name as 802.16 in September 2004 . In this version, the whole family of the standard is configured and approved. These amendments were included in the current applicable version of standard IEEE 802.16 on December 2005[4-5]. So in this version the PHY and MAC layer were combined together which results in enhancement of fixed and mobile operation in licensed band. 


\section{SIMULATION MODEL}

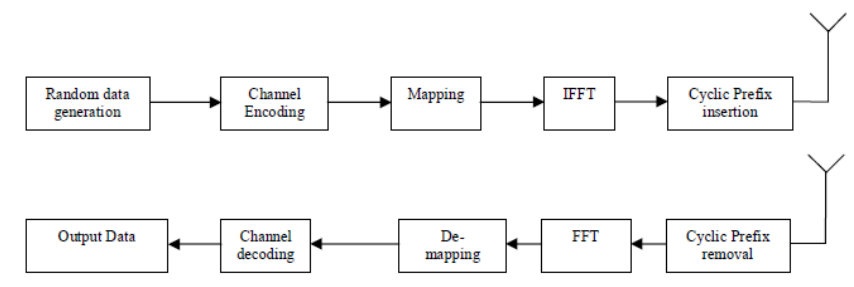

Fig 1: A Block diagram representation of the transmitter and receiver of WiMAX Physical Layer.

The structure of the baseband part of the transmitter and the physical layer of the IEEE 802.16 Wireless MAN-OFDM receiver is shown in Figure 1. This structure corresponds to air interface. In this setup, we have implemented the essential features of the specification namely random data generation, channel encoding, mapping and cyclic prefix rate.

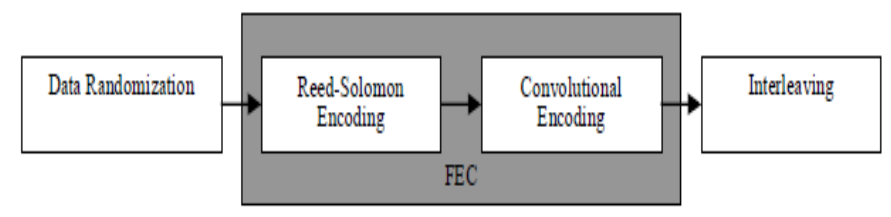

Fig 2: channel encoding setup [7]

Second block of transmitter is channel coding which composed of three steps namely Randomization, Forward Error Correction (FEC) and interleaving. FEC is done in two phases through the outer Reed-Solomon (RS) and inner Convolution Code (CC) as shown in figure 2 .

The forward error control (FEC) consists of a Reed-Solomon (RS) outer code and a rate compatible Convolutional Code (CC) inner code. A block Reed Solomon $(255,239,8)$ code based on the Galois field GF (28) with a symbol size of 8 bits is chosen such that it processes a block of 239 symbols and simultaneously can correct up to 8 symbols. [7]

Reed Solomon Encoder is used to encapsulate the data with coding blocks. These coding blocks are helpful in dealing with the burst errors. The block formatted (Reed Solomon encoded) data stream is then passed through a convolutional interleaver. The convolutionally encoded bits are interleaved at its output. In this set up we used three complex modulation schemes such as QPSK, 16- QAM, and 64-QAM modulation and finally fed to an OFDM modulator for transmission.

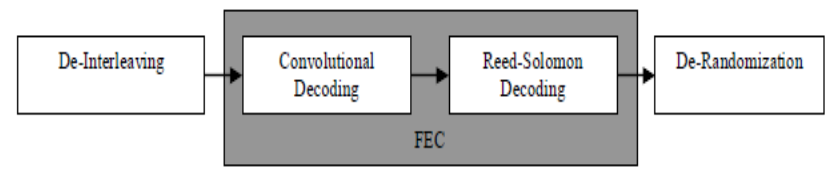

Fig 3: Channel decoding system [7]
While receiver blocks are basically the inverse of the transmitter blocks. At the receiving side, a reverse process (including deinterleaving and decoding) is executed to obtain the original data bits. The de-interleaving process only changes the order of received data and the error probability remains intact. In the $\mathrm{CC}$ decoder and the RS-decoder stage some errors may be corrected which results in lower error rates as illustrate in figure 3.

\section{STANFORD UNIVERSITY INTERIM (SUI)} CHANNEL

In our investigation six channels are used to address three different terrains. These three terrains are defined as A, B and C. A is hilly terrain with heavy tree density, B is hilly terrain with light tree density or flat terrain with moderate to heavy tree density and $\mathrm{C}$ is mostly flat terrain with light tree density. In SUI propagation model parameters are dependent upon terrain, tree density, antenna height and beam width, season or time of the year wind speed.

For A-type, a hilly topology with a high foliage density is considered, while for C-type terrain a mostly flat environment with low foliage density is considered. Lastly, B-type terrains cover a medium foliage density as well as a moderate hilly ground.

These models can be used for simulation, design, development and testing of technologies suitable for fixed broadband wireless applications. The following parameters were selected on the basis of some statistical models. Schematic view of all the six channels is as shown in table 1.

Table 1: Terrain Types for SUI Channel

\begin{tabular}{|l|l|}
\hline Terrain Type & SUI Channels \\
\hline $\begin{array}{l}\text { C (Flat terrain with light } \\
\text { tree density) }\end{array}$ & SUI-1, SUI-2 \\
\hline $\begin{array}{l}\text { B (Hilly terrain with light } \\
\text { tree density) }\end{array}$ & SUI-3, SUI-4 \\
\hline $\begin{array}{l}\text { A (Hilly terrain with } \\
\text { heavy tree density) }\end{array}$ & SUI-5, SUI-6 \\
\hline
\end{tabular}

\section{SIMULATION RESULTS AND DISCUSSION OF}

\section{SUI}

In this section, we have simulated Bit Error Rate versus Signal to Noise Ratio of WiMAX system with SUI Channels by varying the high M-ary modulation and different cyclic prefix rate. For this set up, we have used MATLAB software for simulation purposes. The parameters initially adjusted for simulation are (a) number of simulated OFDM symbols (b) Cyclic Prefix length (c) modulation (d) coding rate (e) range of SNR values (f) various channel models. Firstly, input data stream is randomly generated. Each single block of the 
transmitter is tested with respect to the receiver side to confirm that each block works perfectly. The performance evaluation of system is analyzed with BER performance under different channel conditions and varying parameters as mentioned above. [6-9].

Figure 4 to 7 shows the performance of WiMAX IEEE 802.16 standard under SUI-1 Channel for cyclic prefix rate 1/4, 1/8, $1 / 16,1 / 32$. In this simulation QPSK, 16-QAM and 64-QAM modulation formats are used with different coding rate such as $1 / 2$ and $3 / 4$.

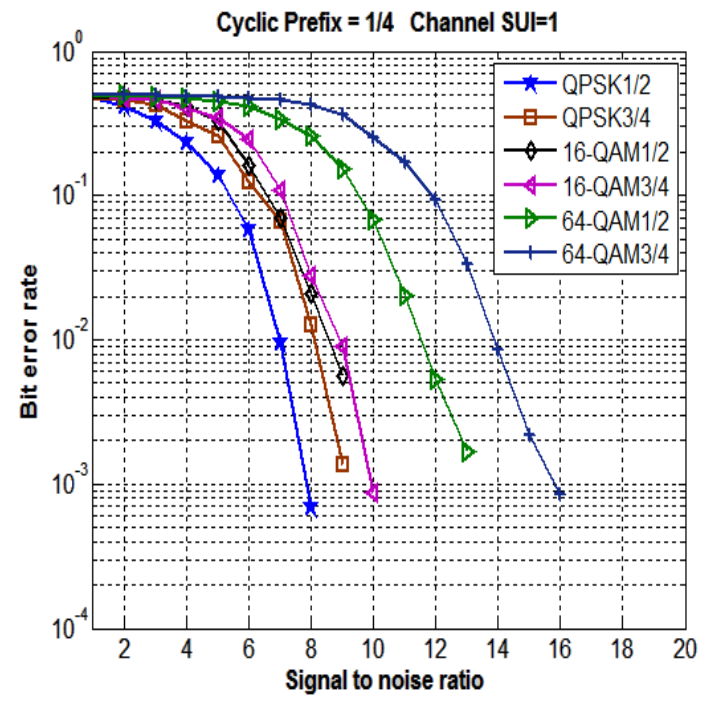

Fig 4: System performance under SUI-1 channel at different modulation schemes for Convolutional Encoder with 1/2 and 3/4 code rate at fixed Cyclic Prefix 1/4.

Figure 4 illustrate performance of system under SUI-1 channel for different modulation schemes. It shows the effect of SNR on BER. From the graph it is clear that less SNR in case of QPSK1/2 modulation schemes is required than the 64-QAM3/4 for same bit rate. The required SNR for same BER with different modulation schemes such as QPSK 1/2, QPSK3/4, 16-QAM1/2, 16-QAM3/4, 64-QAM1/2 and 64-QAM3/4 goes on increasing as shown in figure 4.

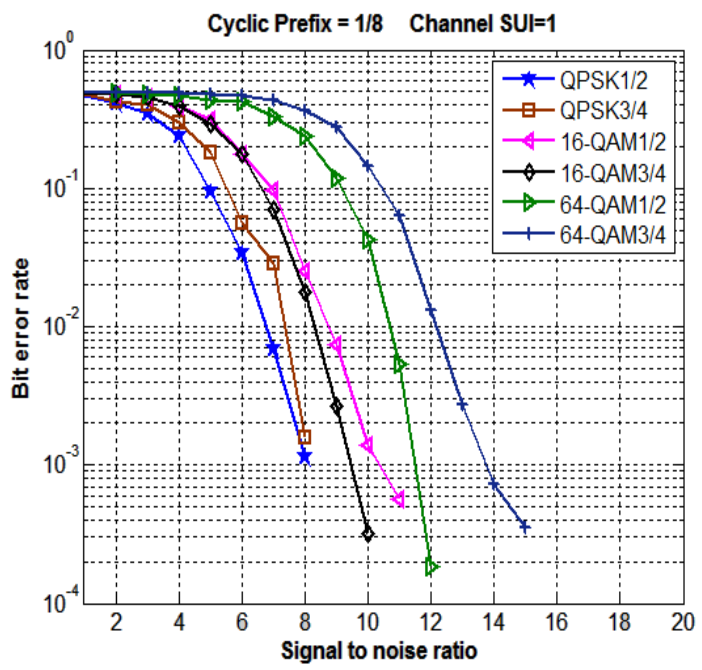

Fig 5: System performance under SUI-1 channel at different modulation schemes for Convolutional Encoder with 1/2 and 3/4 code rate at fixed Cyclic Prefix 1/8.

From figure 5 it is observed that for SUI-1 channel the SNR performance to achieve the BER 10-3 of convolutional 1/2 code rate for QPSK modulation technique is better than all other modulation techniques.

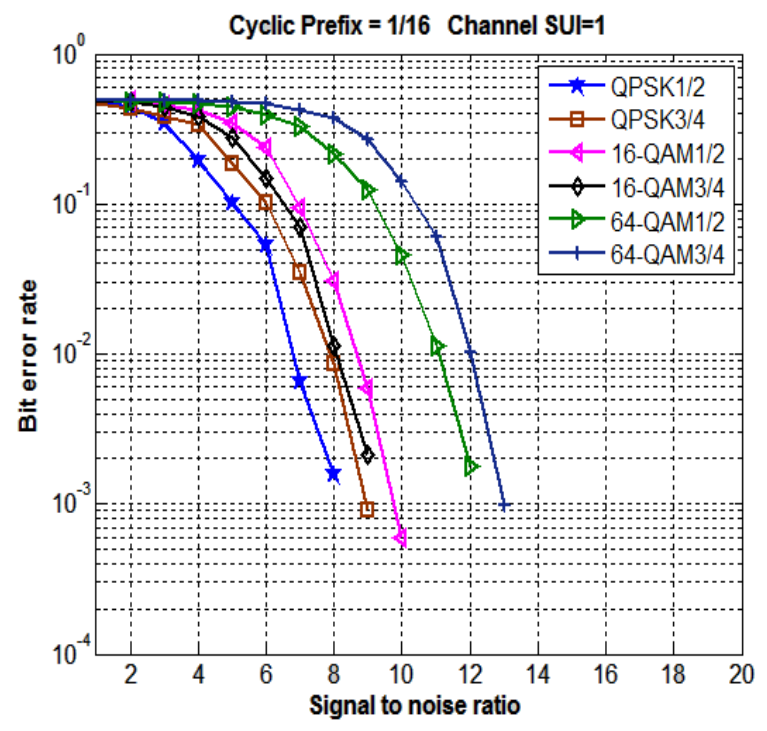

Fig 6: System performance under SUI-1 channel at different modulation schemes for Convolutional Encoder with 1/2 and 3/4 code rate at fixed Cyclic Prefix 1/16.

From figure 6 it is observed that for SUI-1 channel even though a high SNR is required for 64-QAM with coding rate $1 / 2$ but still it is used to carry the more no. of bits in one symbol which results in the improvement of Spectral efficiency of WiMAX system. 


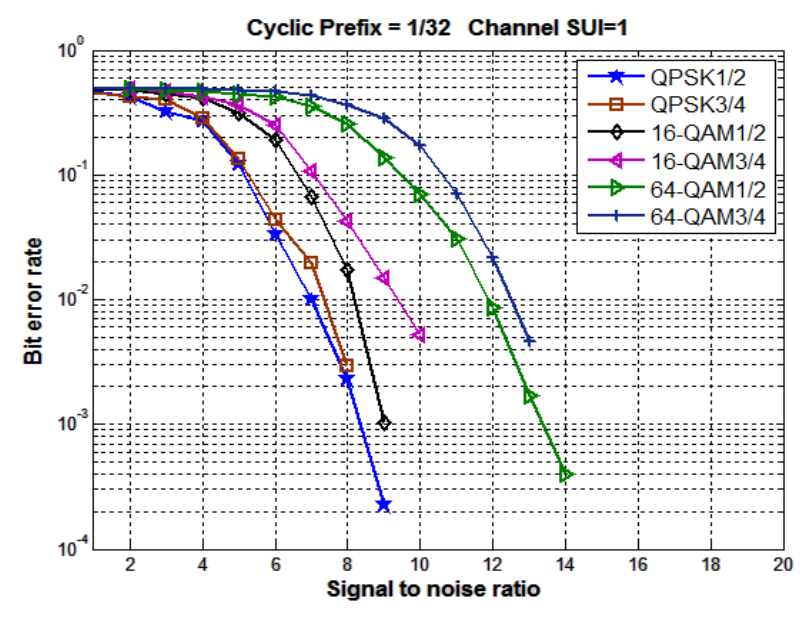

Fig 7: System performance under SUI-1 channel at different modulation schemes for Convolutional Encoder with 1/2 and 3/4 code rate at fixed Cyclic Prefix 1/32.

Figure 7 illustrate performance of system under SUI-1 channel for different modulation schemes. It shows that QPSK with code rate $1 / 2$ and $3 / 4$, transmission is best for $1 / 2$. But generally in convolutional code $1 / 2$ every two bit transmitted one is useful and other is redundant.

Table 2: Performance Evaluation in terms of SNR under SUI-1 Channel to achieve the BER at 10-3

\begin{tabular}{|l|l|l|l|l|l|l|}
\hline $\mathrm{CP}$ & $\begin{array}{l}\text { QPSK } \\
1 / 2\end{array}$ & $\begin{array}{l}\text { QPSK } \\
3 / 4\end{array}$ & $\begin{array}{l}16 \\
\text { QAM } \\
1 / 2\end{array}$ & $\begin{array}{l}16 \\
\text { QAM } \\
3 / 4\end{array}$ & $\begin{array}{l}64 \\
\text { QAM } \\
1 / 2\end{array}$ & $\begin{array}{l}64 \\
\text { QAM } \\
3 / 4\end{array}$ \\
\hline $1 / 4$ & $8 \mathrm{~dB}$ & $9 \mathrm{~dB}$ & $10 \mathrm{~dB}$ & $10 \mathrm{~dB}$ & $13 \mathrm{~dB}$ & $16 \mathrm{~dB}$ \\
\hline $1 / 8$ & $8 \mathrm{~dB}$ & $9 \mathrm{~dB}$ & $10 \mathrm{~dB}$ & $10 \mathrm{~dB}$ & $12 \mathrm{~dB}$ & $14 \mathrm{~dB}$ \\
\hline $\begin{array}{l}1 / 1 \\
6\end{array}$ & $9 \mathrm{~dB}$ & $9 \mathrm{~dB}$ & $10 \mathrm{~dB}$ & $10 \mathrm{~dB}$ & $13 \mathrm{~dB}$ & $13 \mathrm{~dB}$ \\
\hline $\begin{array}{l}1 / 3 \\
2\end{array}$ & $9 \mathrm{~dB}$ & $9 \mathrm{~dB}$ & $9 \mathrm{~dB}$ & $11 \mathrm{~dB}$ & $14 \mathrm{~dB}$ & $15 \mathrm{~dB}$ \\
\hline
\end{tabular}

Table 2 shows the comparative analysis of Signal to Noise Ratio in different modulation format. It is observed from the table 2 that as we compare all the cyclic prefixes. It further shows that cyclic prefix $1 / 4$ reduces the SNR in better way as compared to other cyclic prefix rates $1 / 8,1 / 16,1 / 32$. So it can be observed from above discussion that $1 / 4$ cyclic prefix can completely eliminate ISI and avoids channel delay spread.

\section{RESULT AND DISCUSSION FOR SUI-2 CHANNEL UNDER THE DIFFERENT} MODULATION SCHEMES

Figure 8 to 11 shows the performance of WiMAX IEEE 802.16 standard under SUI-2 Channel for cyclic prefix rate 1/4, 1/8, 1/16, 1/32. In this simulation QPSK, 16-QAM and 64-QAM modulation formats are used with different coding rate such as $1 / 2$ and $3 / 4$.

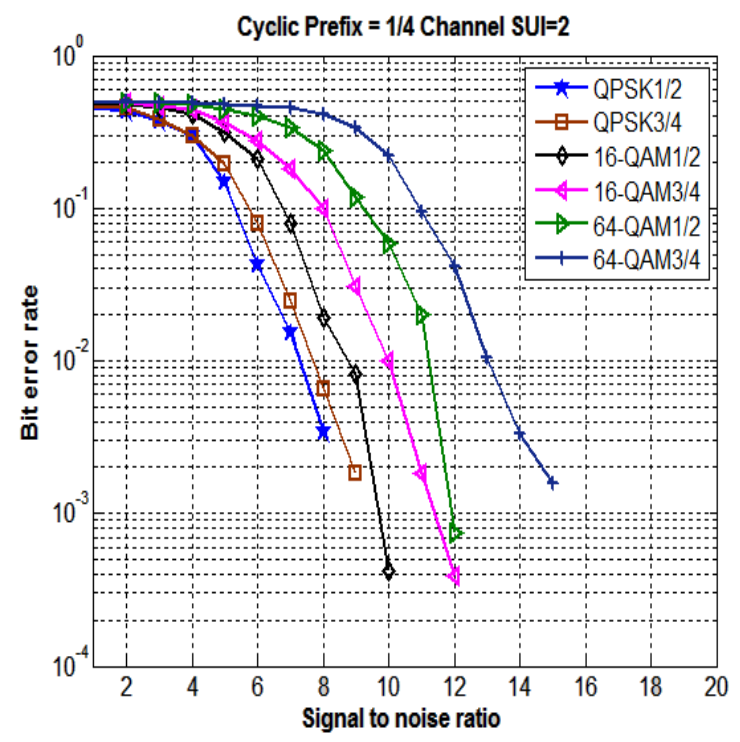

Fig 8: System performance under SUI-2 channel at different modulation schemes for Convolutional Encoder with 1/2 and 3/4 code rate at fixed Cyclic Prefix 1/4.

We observed here the performance of SUI-2 channel at different modulation schemes under the different code rate1/2 and 3/4 for fixed cyclic prefix 1/4. Graphical representation of BER versos SNR for different modulations scheme is illustrated in figure 8 . From figure 8 it is observed that SNR required to achieve the desired BER value (10-3) with QPSK 1/2 code rate is less than all other modulation techniques.

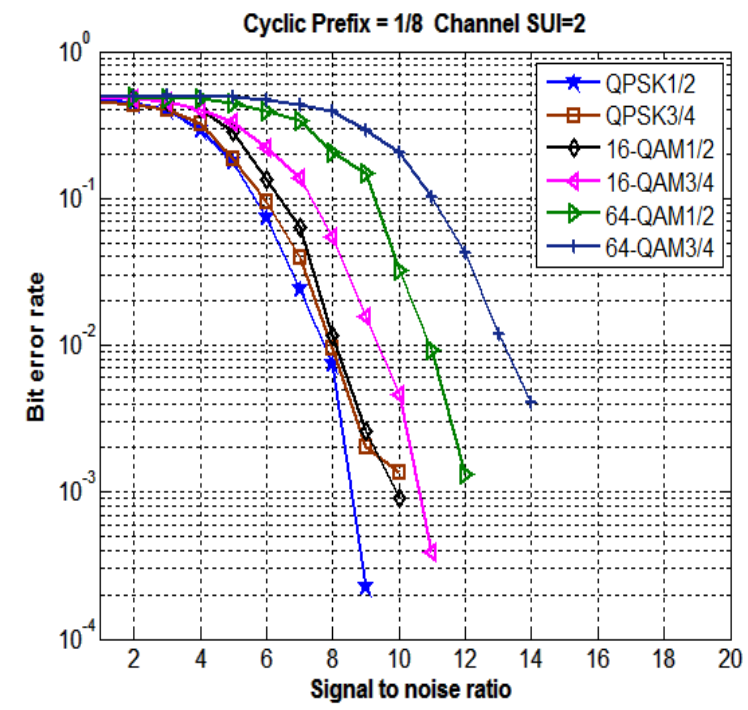

Fig 9: System performance under SUI-2 channel at different modulation schemes for Convolutional Encoder with 1/2 and 3/4 code rate at fixed Cyclic Prefix 1/8. 
Figure 9 shows the quantized analysis of Signal to Noise Ratio under SUI-2 channel, the performance of the system under QPSK modulation in $1 / 2$ convolutional code rate is quite satisfactory as compared to other modulation techniques.

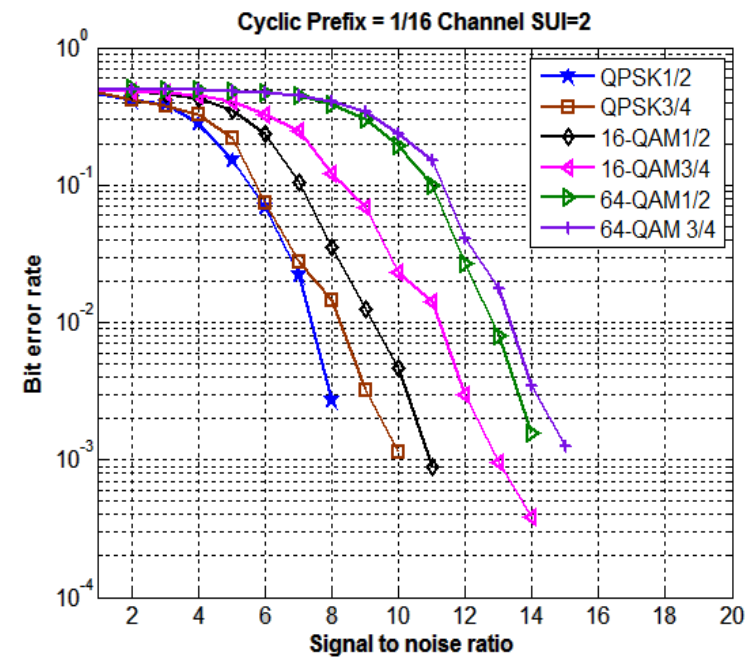

Fig 10: System performance under SUI-2 channel at different modulation schemes for Convolutional Encoder with 1/2 and 3/4 code rate at fixed Cyclic Prefix 1/16.

Figure 10 shows the quantized analysis of Signal to Noise Ratio under SUI-2 channel. In this case the system shows better performance with less SNR required under the various modulation schemes. So QPSK modulation with $1 / 2$ convolutional code rate give us more satisfactory performance as compared to other modulation techniques.

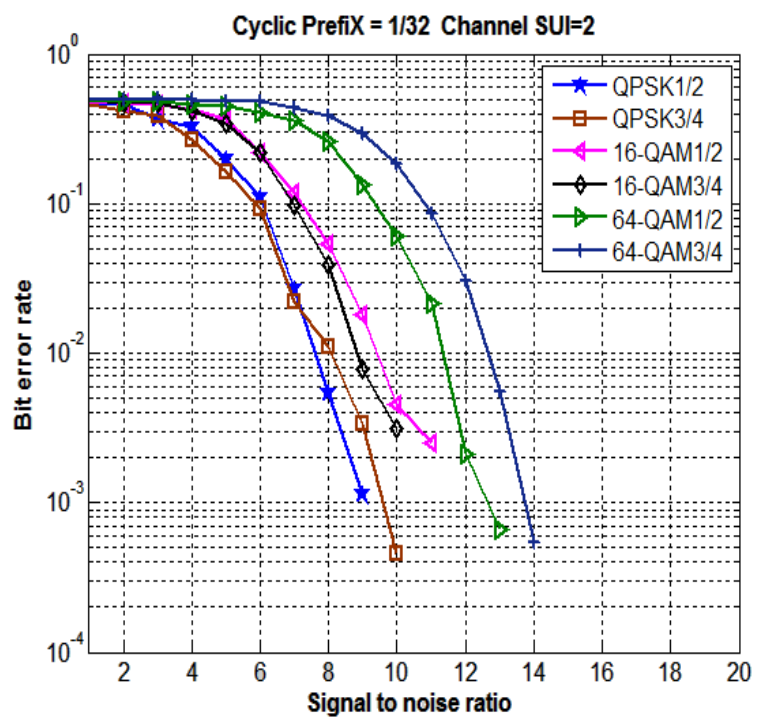

Fig 11: System performance under SUI-2 channel at different modulation schemes for Convolutional Encoder with 1/2 and 3/4 code rate at fixed Cyclic Prefix 1/32.
Figure 11 shows the quantized analysis of Signal to Noise Ratio with SUI-2 channel under the different modulation format. The result in this figure reveals that at the lower modulation QPSK $1 / 2$ provides better performance with less SNR.

Table 3: Tabular representation of SUI-2 channel in terms of SNR under SUI-2 Channel to achieve the BER at 10-3 is shown below:

\begin{tabular}{|l|l|l|l|l|l|l|}
\hline $\mathrm{CP}$ & $\begin{array}{l}\text { QPSK } \\
1 / 2\end{array}$ & $\begin{array}{l}\text { QPSK } \\
3 / 4\end{array}$ & $\begin{array}{l}16 \\
\text { QAM } \\
1 / 2\end{array}$ & $\begin{array}{l}16 \\
\text { QAM } \\
3 / 4\end{array}$ & $\begin{array}{l}64 \\
\text { QAM } \\
1 / 2\end{array}$ & $\begin{array}{l}64 \\
\text { QAM } \\
3 / 4\end{array}$ \\
\hline $1 / 4$ & $9 \mathrm{~dB}$ & $9 \mathrm{~dB}$ & $10 \mathrm{~dB}$ & $11 \mathrm{~dB}$ & $12 \mathrm{~dB}$ & $15 \mathrm{~dB}$ \\
\hline $1 / 8$ & $9 \mathrm{~dB}$ & $9 \mathrm{~dB}$ & $10 \mathrm{~dB}$ & $11 \mathrm{~dB}$ & $13 \mathrm{~dB}$ & $15 \mathrm{~dB}$ \\
\hline $1 / 16$ & $9 \mathrm{~dB}$ & $10 \mathrm{~dB}$ & $11 \mathrm{~dB}$ & $13 \mathrm{~dB}$ & $14 \mathrm{~dB}$ & $15 \mathrm{~dB}$ \\
\hline $1 / 32$ & $9 \mathrm{~dB}$ & $10 \mathrm{~dB}$ & $11 \mathrm{~dB}$ & $13 \mathrm{~dB}$ & $14 \mathrm{~dB}$ & $15 \mathrm{~dB}$ \\
\hline
\end{tabular}

Table 3 shows the comparative analysis of Signal to Noise Ratio in different modulation format. It is observed from the table as we decrease the cyclic prefix from $25 \%(1 / 4)$ the required SNR increases. From the different tabular entries it is clear that cyclic prefix 1/4 required less SNR. Large cyclic prefix signifies large time gap between two frames. This Large value of cyclic prefix gives extra time to receive signal from multipath signals.

\section{RESULT AND DISCUSSION FOR SUI-3 CHANNEL UNDER THE DIFFERENT MODULATION SCHEMES}

Figure 12 to 15 shows the performance of WiMAX IEEE 802.16 standard under SUI-3 Channel for cyclic prefix rate 1/4, 1/8, 1/16, 1/32. In this simulation QPSK, 16-QAM and 64-QAM modulation formats are used with different coding rate such as $1 / 2$ and $3 / 4$.

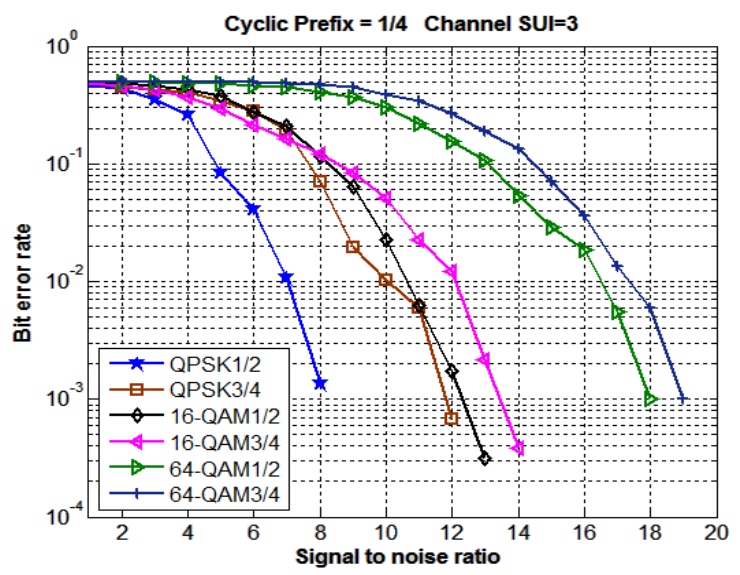

Fig 12: System performance under SUI-3 channel at different modulation schemes for Convolutional Encoder with 1/2 and 3/4 code rate at fixed Cyclic Prefix 1/4. 
Figure 12 illustrate performance of system under SUI-1 channel for different modulation schemes. From the graph it is clear that the required SNR to achieve BER (10-3) in case of QPSK1/2 modulation schemes is less than the 64-QAM3/4.

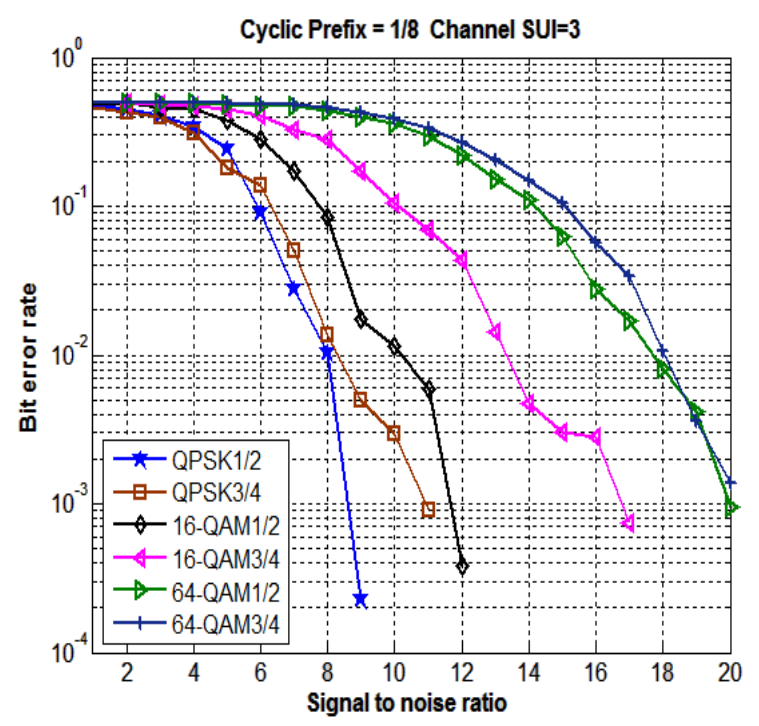

Fig 13: System performance under SUI-3 channel at different modulation schemes for Convolutional Encoder with 1/2 and 3/4 code rate at fixed Cyclic Prefix 1/8

From figure 13 it is observed when we use $1 / 2$ coding rate the required SNR for QPSK is approximately 9dB to achieve 10-3 BER. Similarly when $3 / 4$ coding rate is used the required SNR is approximately $11 \mathrm{~dB}$ for QPSK. Hence, QPSK 1/2 shows better performance than other format of modulation schemes in order to achieve 10-3 BER.

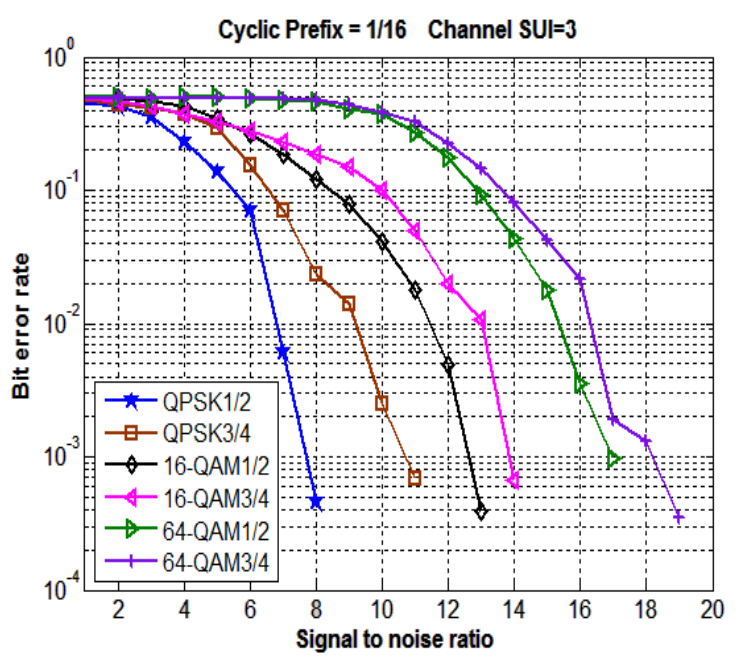

Fig 14: System performance under SUI-3 channel at different modulation schemes for Convolutional Encoder with 1/2 and 3/4 code rate at fixed Cyclic Prefix 1/16.
Figure 14 shows the quantized analysis of Signal to Noise Ratio with SUI-3 channel, it is observed from the figure as we decrease the cyclic prefix rate from $25 \%(1 / 4)$ the SNR requirement increases for 64-QAM modulation with code rate $3 / 4$.

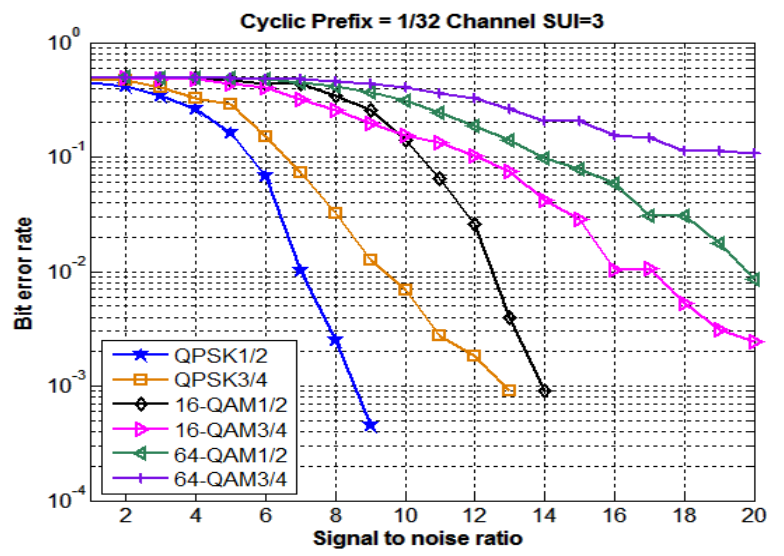

Fig 15: System performance under SUI-3 channel at different modulation schemes for Convolutional Encoder with 1/2 and 3/4 code rate at fixed Cyclic Prefix 1/32.

Figure 15 shows that, 64-QAM modulation at coding rate $1 / 2$ and 3/4 achieve the BER (10-3) the SNR required increased by $20 \mathrm{~dB}$ than the desired value. At low SNR fading is more and signal strength is going low as distance increases, which further degrade the performance of system. To overcome this problem we choose higher value of cyclic prefix. Large cyclic prefix means large time gap between two frames. A large value gives extra time to receive signal from multipath signals.

\section{ADDITIVE WHITE GAUSSIAN NOISE (AWGN)}

The simplest radio environment in which a wireless communications system or a local positioning system or proximity detector based on Time of- flight will have to operate is the Additive-White Gaussian Noise environment. Additive white Gaussian noise (AWGN) is the commonly used to transmit signal while signals travel from the channel and simulate background noise of channel. The mathematical expression in received signal $\mathrm{r}(\mathrm{t})=\mathrm{s}(\mathrm{t})+\mathrm{n}(\mathrm{t})$ that passed through the AWGN channel where $s(t)$ is transmitted signal and $n(t)$ is background noise shown in figure 16.

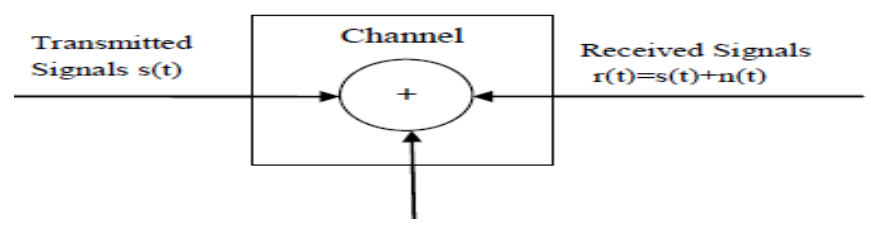

Fig 16: Block diagram of AWGN channel model 


\section{RESULTS AND DISCUSSION OF AWGN}

\section{CHANNEL}

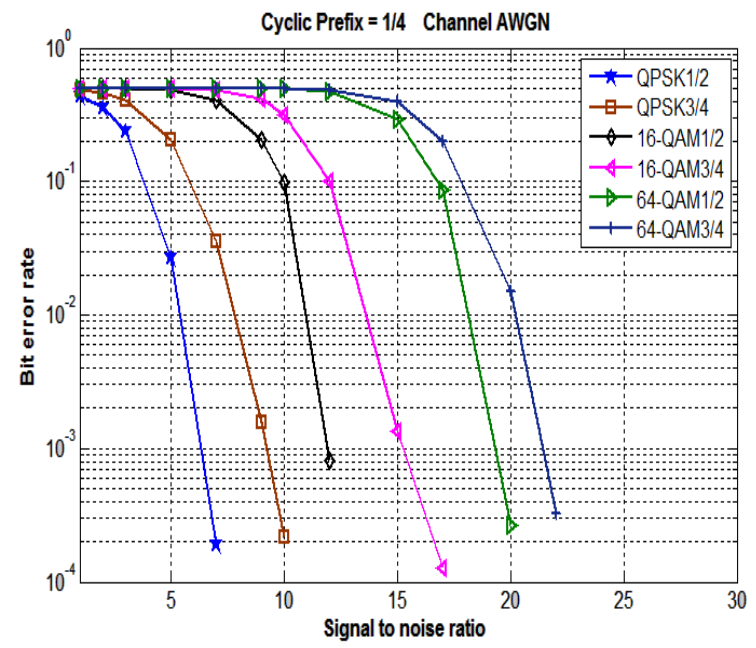

Fig 17: System performance under AWGN channel at different modulation schemes for Convolutional Encoder with 1/2 and 3/4 code rate at fixed Cyclic Prefix 1/4.

From figure 17 it is observed when we use $1 / 2$ coding rate the required SNR for QPSK is approximately 6dB to achieve 10-3 BER. Similarly when $3 / 4$ coding rate is used the required SNR is approximately 10dB for QPSK. Hence, QPSK 1/2 shows better performance than other format of modulation schemes in order to achieve 10-3 BER.

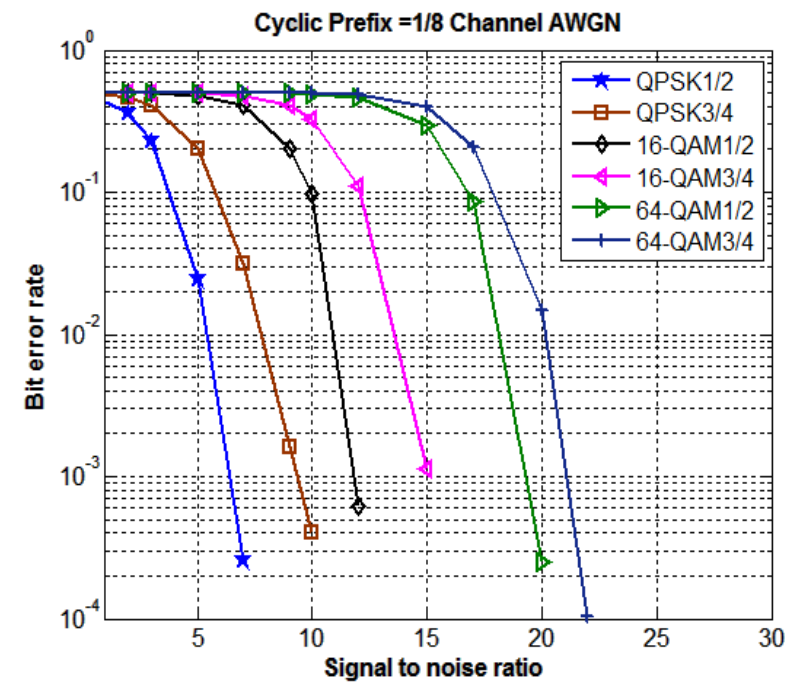

Fig 18: System performance under AWGN channel at different modulation schemes for Convolutional Encoder with 1/2 and 3/4 code rate at fixed Cyclic Prefix 1/8.
Figure 18 has shown the quantized analysis of Signal to Noise Ratio with SUI-3 channel. The result in this figures reveal that the various modulation and coding scheme $1 / 2$ provides better performance with less SNR.

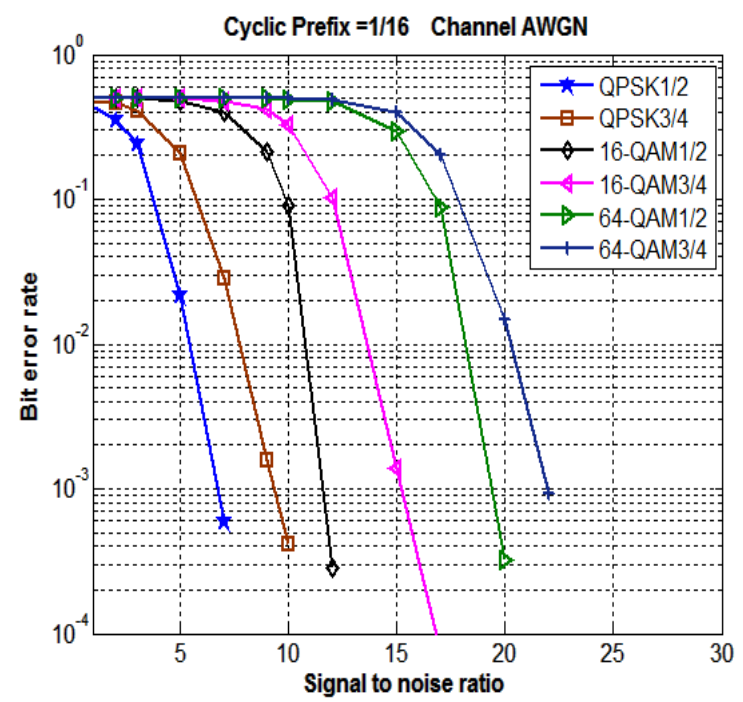

Fig 19: System performance under AWGN channel at different modulation schemes for Convolutional Encoder with 1/2 and 3/4 code rate at fixed Cyclic Prefix 1/16.

Figure 19 has shows that, high SNR is required for 64-QAM with coding rate $1 / 2$ but still this is used to carry the more no. of bits in one symbol which results in the improvement of Spectral efficiency of WiMAX system. The SNR for different modulation schemes such as QPSK 1/2, QPSK3/4, 16-QAM1/2, 16QAM3/4, 64-QAM1/2 and 64-QAM3/4 goes on increasing as shown in different curves of figure 19.

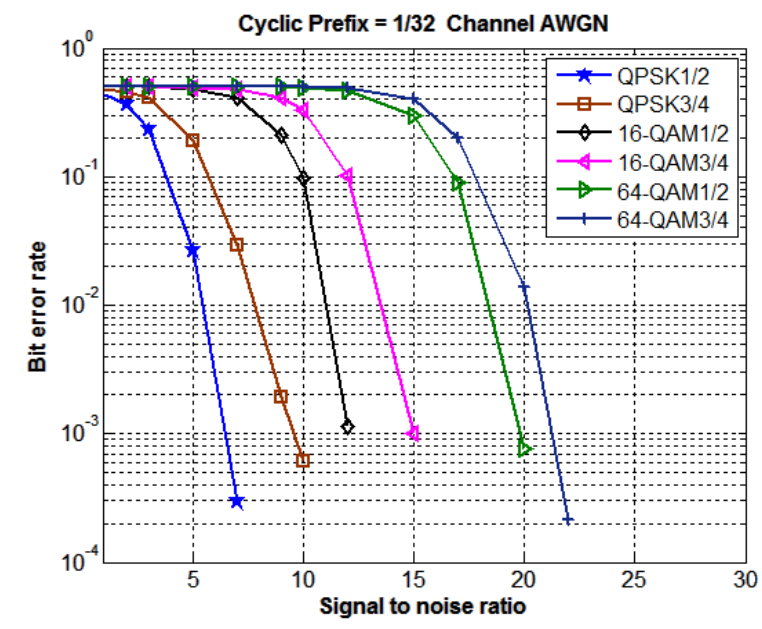

Fig 20: System performance under AWGN channel at different modulation schemes for Convolutional Encoder with 1/2 and 3/4 code rate at fixed Cyclic Prefix 1/32. 
Figure 20 has shown the quantized analysis of Signal to Noise Ratio with SUI-3 channel; from the graph it is clear that SNR required in case of QPSK1/2 modulation schemes is less than the 64-QAM3/4.

\section{CONCLUSIONS}

We implemented WiMAX IEEE 802.16 physical layer using MATLAB in order to evaluate the performance under various channel models. The result for various BER versus SNR plots for the high $\mathrm{M}$-ary modulation techniques with different cyclic prefix rate $(1 / 4,1 / 8,1 / 16,1 / 32)$, under AWGN, SUI 1, SUI 2, and SUI 3 channel model. The results reveal that the various modulation and coding scheme provides better performance with less SNR at Cyclic Prefix rate 1/4. However, 64-QAM may be used to increase the spectral efficiency with higher SNR required to achieve the BER at 10-3.

\section{REFERENCES}

[1] Ebian, M. Shokair, and K. H. Awadalla, "Performance Evaluation of WiMAX System Using Convolution Product Code (Cpc)", Progress in electromagnetics Research, Vol. 5, 125-133, 2008.

[2] Md. Ashraful Islam, Md.Zahid Hagan, "Performance Evaluation of WiMAX physical layer under Adaptive Modulation Techniques And Communication Channels", (IJCSIS) International Journal of Computer Science and Information Security, Vol. 5, no.1, pp 111-114, 2009.

[3] Maninder Singh, R.S. Uppal, Jaspal Singh, "WiMAX with different Modulation Techniques and Code Rates" International Journal of Information and Telecommunication Technology, vol. 3, no.1, pp.1-5, Feb 2011.

[4] Mohammad Sfiqul Islam, Gouri Rani Barai, Atiq Mahmood, "Performance analysis of different modulation schemes using OFDM techniques in Rayleigh fading channel", International Journal of Fundamental Physical Sciences vol.1, no.1, pp.22-27 March-2011.

[5] Pratyush Sharma, Abhishek Sharma, Kailash C. Bandhu, "Performance Evaluation of Adaptive Modulation Techniques and offered Load in OFDM-based WiMAX Network by Considering Cyclic Prefix", International Journal of Scientific \& Engineering Research, vol.2, no., pp.1-5 Nov-2011.

[6] Shanar Askar, Shahzad Memon, M.S Kalhoro, “ Impact of modeling different Fading Channel on Wireless MAN Fixed IEEE802.16d OFDM System with Diversity Transmission Technique", world Academy of science, Engineering and Technology, vol. no.1, pp.1473-1478, 2011.

[7] K.Chiranjeevi, G.B.S.R.Naidu, Prabhakar Telagarapu, "Analysis of Coding Techniques in WiMAX", International Journal of Computer Application, vol. 22, no. 3, pp.19-26 May 2011.

[8] Omar Arafat, K.Dimyati, "Performance Parameter of Mobile WiMAX: A Study on the Physical layer of
Mobile WiMAX under Different Communication Channels \& Modulation Technique" IEEE 2010 Second International Conference on Computer Engineering and Applications. vol. No. 1, pp.553-537.

[9] Mohamed A. Mohamed, Heba M.Abd-El-Atty, "Performance Simulation of IEEE 802.16e WiMAX Physical Layer”, 2010 IEEE. vol. No. 2, pp.1-8. 